\title{
Rigid isotopy of maximally writhed links
}

\author{
Grigory Mikhalkin and Stepan Orevkov
}

\begin{abstract}
This is a sequel to our paper [Maximally writhed real algebraic links, Invent. Math. 216 (2019), no. 1, 125-152], which identified maximally writhed algebraic links in $\mathbb{R P}^{3}$ and classified them topologically. In this paper, we prove that all maximally writhed links of the same topological type are rigidly isotopic; that is, one can be deformed into another with a family of smooth real algebraic links of the same degree.
\end{abstract}

\section{Introduction}

A real algebraic curve $A \subset \mathbb{P}^{3}$ is a (complex) 1-dimensional subvariety invariant with respect to the involution of complex conjugation conj: $\left(z_{0}: z_{1}: z_{2}: z_{3}\right) \mapsto\left(\bar{z}_{0}: \bar{z}_{1}: \bar{z}_{2}: \bar{z}_{3}\right)$. We denote by $\mathbb{R} A$ the fixed point locus of conj $\left.\right|_{A}$; note that $\mathbb{R} A=A \cap \mathbb{R P}^{3}$. Following the classical terminology, by a real branch of $A$, we mean the image $\nu(K)$ for a connected component $K$ of $\mathbb{R} \tilde{A}$, where $\nu: \tilde{A} \rightarrow A$ is a normalization.

In the case of non-singular $A$, we call $L=\mathbb{R} A$ the real algebraic link, cf. [MO19], if every component of the normalization of $A$ has non-empty real part and if $L$ is not contained in any proper projective subspace of $\mathbb{R P}^{3}$. We say that $L$ is irreducible if $A$ is irreducible. In this paper, real algebraic links as well as algebraic curves are assumed to be irreducible.

The degree $d \in \mathbb{Z}$ of a real algebraic link $L \subset \mathbb{R P}^{3}$ is a positive number such that $[A]=$ $d\left[\mathbb{P}^{1}\right] \in H_{2}\left(\mathbb{P}^{3}\right) \cong \mathbb{Z}$; the genus of $L$ is the genus of $A$. Given a point $p \in \mathbb{R} \mathbb{P}^{3} \backslash \mathbb{R} A$, we denote by $\pi_{p}: \mathbb{P}^{3} \backslash\{p\} \rightarrow \mathbb{P}^{2}$ the linear projection from $p$. The image $\pi_{p}(A) \subset \mathbb{P}^{2}$ is a plane curve of the same degree $d$. If $p$ is chosen generically, then $\pi_{p}(A)$ is a nodal curve. The set

$$
\left(\pi_{p}(A) \cap \mathbb{R P}^{2}\right) \backslash \pi_{p}(\mathbb{R} A)
$$

is not always empty. It is a finite set consisting of solitary nodes of $\mathbb{R} \pi_{p}(A)=\pi_{p}(A) \cap \mathbb{R P}^{2}$, that is, the points of real intersection of pairs of complex conjugate local branches. It was shown in [Vir01] that each node of $\mathbb{R} \pi_{p}(A)$ may be prescribed a sign \pm 1 or 0 so that the sum $w(L)$ of the signs of all nodes does not depend on a choice of $p$ and is an invariant of $L$.

Non-solitary nodes of $\mathbb{R} \pi_{p}(A)$ are intersection points of pairs of real local branches of $A$. If these local branches belong to different real branches of $A$, then the sign of the corresponding node is defined as zero. Otherwise, the definition of the sign at a non-solitary node agrees with the convention used in knot theory for the definition of the writhe of the knot diagram; see

Received 24 February 2019, accepted in final form 7 August 2020.

2020 Mathematics Subject Classification 14P25, $14 \mathrm{H} 50$.

Keywords: real algebraic links, rigid isotopy, encomplexed writhe, maximally writhed links.

This journal is (C) Foundation Compositio Mathematica 2021. This article is distributed with Open Access under the terms of the Creative Commons Attribution Non-Commercial License, which permits non-commercial reuse, distribution, and reproduction in any medium, provided that the original work is properly cited. For commercial re-use, please contact the Foundation Compositio Mathematica.

The research for this paper was supported in part by the grants 178828, 182111 and the NCCR SwissMAP project of the Swiss National Science Foundation (G.M.), and by RFBR grant No 17-01-00592a (S.O.). 


\section{RIGID ISOTOPY OF MAXIMALLY WRITHED LINKS}

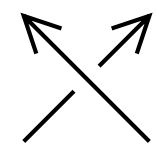

$-1$

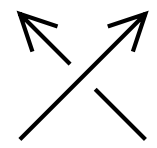

$+1$

FiguRE 1. Writhe signs for a crossing point of two real branches on the knot diagram

Figure 1 (we choose any orientations of the two local branches that can agree with an orientation of the real branch containing them).

In the case of solitary nodes, the signs were introduced in [Vir01]. Accordingly, $w(L)$ is called the encomplexed writhe (as in [Vir01]) or the Viro invariant of $L$. Note that the number of nodes of $\pi_{p}(A)$ is $N_{d}-g$, where $N_{d}=(d-1)(d-2) / 2$. Thus, we have the straightforward upper bound

$$
|w(L)| \leqslant N_{d}-g \leqslant N_{d}
$$

for any real algebraic link $L \subset \mathbb{R P}^{3}$ of degree $d$. Accordingly, an (irreducible) real algebraic link of degree $d$ with $|w(L)|=N_{d}$ is called a maximally writhed knot or an $M W$-knot (since this property implies that $g=0$, the curve $A$ is rational and thus $L$ is connected).

The following extremal property of (1.1) was shown in [MO18].

Theorem 1 ([MO18]). If $L, L^{\prime} \subset \mathbb{R P}^{3}$ are $M W$-knots of the same degree, then the pairs $\left(\mathbb{R P}^{3}, L\right)$ and $\left(\mathbb{R} \mathbb{P}^{3}, L^{\prime}\right)$ are diffeomorphic.

In other words, the topological type of an $M W$-knot of a given degree is unique.

Now, consider the case of an arbitrary $g$. By Harnack's inequality, the number of real branches of $A$ is at most $g+1$. The (irreducible) real curves $A$ with $g+1$ real branches are known as $M$-curves. In this case, the complement $A \backslash \mathbb{R} A$ consists of two components bounded by $\mathbb{R} A$. The orientations of two component of $A \backslash \mathbb{R} A$ yield a pair of opposite orientations of $L$. Through these orientations, an orientation of one real branch of $A$ determines the orientation of all other real branches, and we also define the sign $\sigma(q)= \pm 1$ in the case when a node $q \in \mathbb{R} \pi_{p}(A)$ corresponds to a crossing point of different real branches of $A$ according to Figure 1 . We set

$$
w_{\lambda}(L)=w(L)+\sum_{q} \sigma(q)=w(L)+2 \sum_{K \neq K^{\prime}} \operatorname{lk}\left(K, K^{\prime}\right)
$$

see [MO19]. Here the first sum is taken over the nodes $q$ corresponding to intersection points of different real branches of $A$, while the second sum is taken over unordered pairs of distinct real branches $K, K^{\prime}$.

Clearly, if $\left|w_{\lambda}(L)\right|=N_{d}$, then $L$ consists of a single real branch. Furthermore, the stronger inequality

$$
\left|w_{\lambda}(L)\right| \leqslant N_{d}-g
$$

holds. It has a chance to be sharp even for a multicomponent $L$.

Definition 1.1. A maximally writhed real algebraic link (or an $M W_{\lambda}$-link) $L \subset \mathbb{R P}^{3}$ is a real algebraic link in $g+1$ real branches, for $g \geqslant 0$, such that the irreducible real algebraic curve $A$ with $\mathbb{R} A=L$ is of genus $g$ and that $\left|w_{\lambda}(L)\right|=N_{d}-g$.

Note that $N_{d}>g$ as, otherwise, $A$ must be planar. Therefore, $w_{\lambda}(L) \neq 0$ if $L$ is an $M W_{\lambda}$-link. We refer to the sign of $w_{\lambda}(L)$ as the chirality of an $M W_{\lambda}$-link $L$. 


\section{G. Mikhalkin and S. Orevkov}

Let $\alpha=\left(a_{0}, \ldots, a_{g}\right)$ be a partition of the number $d-2$ into $l=g+1$ positive integer numbers. This means that $a_{0} \geqslant \cdots \geqslant a_{g}$ are positive integer numbers such that $\sum_{j=0}^{g} a_{j}=d-2$. In [MO19], with each $\alpha$, we have associated a (topological) link $W_{g}(\alpha)$ in $g+1$ components. Namely, $W_{g}(\alpha)$ is a link that sits on the boundary (the union of $g+1$ hyperboloids) of a regular neighborhood of the Hopf link $H$ in $g+1$ components. Each of the $g+1$ hyperboloids contains a single component of $W_{g}(\alpha)$. Furthermore, the linking number of the $j$ th component $K_{j}$ (enhanced with some orientation) and the $j$ th component of the Hopf link is $a_{j}+2$, while the linking number of $K_{j}$ with any other component of $H$ is $a_{j}$.

Theorem 2 ([MO19]). For every $M W_{\lambda}$-link $L$ of degree $d$, there exists a partition $\alpha=\left(a_{0}, \ldots, a_{g}\right)$ such that $\left(\mathbb{R P}^{3}, L\right)$ and $\left(\mathbb{R} \mathbb{P}^{3}, W_{g}(\alpha)\right)$ are diffeomorphic.

Conversely, for every $\alpha$, there exists an $M W_{\lambda}$-link $L$ of degree $d$ such that the pairs $\left(\mathbb{R P}^{3}, L\right)$ and $\left(\mathbb{R P}^{3}, W_{g}(\alpha)\right)$ are diffeomorphic.

We say that an $M W_{\lambda}$-link $L$ corresponds to the partition $\alpha$ if $\left(\mathbb{R P}^{3}, L\right)$ and $\left(\mathbb{R P}^{3}, W_{g}(\alpha)\right)$ are diffeomorphic.

The following result is the main theorem of this paper. It strengthens the second half of Theorem 2. Two real algebraic links are called rigidly isotopic if they can be connected with a 1-parameter family of real algebraic links of the same degree.

Theorem 3. If $L$ and $L^{\prime}$ are $M W_{\lambda}$-links of the same chirality corresponding to the same partition $\alpha$, then $L$ and $L^{\prime}$ are rigidly isotopic.

This theorem is a particular case of Theorem 4 (see Section 8), where we describe rigid isotopy classes of nodal curves of any genus. The proof of Theorem 4 is based on the theory of divisors on nodal Riemann surfaces and on the properties of $M W_{\lambda}$-curves established in [MO19].

Note that in the case of rational curves, Theorem 2 corresponds to [MO18, Theorem 1] with a simpler proof specifically for genus 0 . In contrast to that, the only way known to us to prove Theorem 3 is to deduce it from Theorem 4, even in the case of rational curves. In particular, this proof requires [MO19, Theorem 2] in whole generality. Note that Theorem 4 provides another proof of [MO19, Theorem 3], that is, the existence of $M W_{\lambda}$-links isotopic to $W_{g}(\alpha)$ for any partition $\alpha=\left(a_{0}, \ldots, a_{g}\right)$.

\section{Algebraic Hopf links}

Definition 2.1. A real algebraic link $L$ with $l$ real branches is called an irreducible algebraic Hopf link if it is irreducible, its degree is $l+2$, and each real branch of $A$ is non-contractible in $\mathbb{R P}^{3}$.

An irreducible algebraic Hopf link has minimal possible degree among all irreducible real algebraic links with $l$ real branches such that each real branch is non-contractible in $\mathbb{R P}^{3}$ by Proposition 2.2. By Proposition 2.3, it is always a Hopf link topologically.

Proposition 2.2. Let $L \subset \mathbb{R P}^{3}$ be an irreducible real algebraic link of degree $d>1$ such that each component of $L$ is non-contractible in $\mathbb{R P}^{3}$. Then $d \geqslant l+2$.

Proof. Take a pair of conjugate points on $A \backslash \mathbb{R} A \subset \mathbb{P}^{3}$, and consider a real plane through this pair. Since any plane in $\mathbb{R P}^{3}$ must intersect each non-contractible component of $L$, we get at least $l+2$ intersection points. 


\section{RIGID ISOTOPY OF MAXIMALLY WRITHED LINKS}

Proposition 2.3. An irreducible algebraic Hopf link $L$ or its mirror image is an $M W_{\lambda}$-link $W_{l-1}(1, \ldots, 1)$ and thus is topologically isotopic to a Hopf link, that is, the union of $l$ fibers of the (positive) Hopf fibration $\mathbb{R P}^{3} \rightarrow S^{2}$.

Proof. The link $L$ satisfies condition (iiit ${ }^{t}$ ) of [MO19, Theorem 2]. Namely, any plane section in $\mathbb{R} \mathbb{P}^{3}$ intersects $L$ in at least $d-2=l$ points (one for each component). By [MO19, Theorem 2], the link $L$ or its mirror image is topologically isotopic to $W_{l-1}(1, \ldots, 1)$ as $(1, \ldots, 1)$ is the only partition of $l$ into the sum of $l$ positive integers.

Lemma 2.4 (cf. Theorem 2.5 of [Hui03]). An effective real divisor $D=\sum_{j=1}^{n} a_{j} p_{j}$ with $a_{j}>0$ and $p_{j} \in S$ on a real curve $S$ is non-special if at least $g$ distinct real branches of $A$ intersect $\left\{p_{1}, \ldots, p_{n}\right\}$.

Proof. By the Riemann-Roch theorem, a divisor $D$ is special if and only if the difference $K-D$ of the canonical class $K$ and the divisor $D$ is representable with an effective divisor $D^{\prime}$. Suppose that there exists a holomorphic form whose zero divisor is $D+D^{\prime}$. But the zero divisor of a form on each real branch of a curve must be even (if counted with multiplicities). Thus the degree of $D+D^{\prime}$ is at least $2 g$, while the degree of the canonical class is $2 g-2$.

By a real Riemann surface, we mean an (irreducible) Riemann surface $S$ enhanced with an antiholomorphic involution conj. Its real locus $\mathbb{R} S$ is the fixed locus of conj. By Harnack's inequality, $g+1$ is the maximal possible number of real branches of $S$ if $g$ is the genus of $S$. Real curves with this maximality property are known as $M$-curves.

Recall that all effective divisors linearly equivalent to an effective divisor $D$ of degree $d$ form a projective space $|D| \approx \mathbb{P}^{r}$ whose dimension is called the rank $r(D)$ of the divisor. If the divisor is base point free, that is, every point of $S$ is not contained in some divisor from $|D|$, then in addition, we have the map

$$
\iota_{D}: S \rightarrow|D|^{\vee} \approx \mathbb{P}^{r}
$$

to the space $|D|^{\vee}$ projectively dual to $|D|$. The point $x \in S$ is mapped to the hyperplane in $|D|$ consisting of divisors containing $x$. If $S$ is a real Riemann surface and $D$ is a divisor invariant with respect to the conjugation, then we say that $D$ is a real divisor. In this case the map $\iota_{D}$ is also real; that is, it is equivariant with respect to the conjugation and the image $A=\iota_{D}(S) \subset \mathbb{P}^{r}$ is a real projective curve.

Proposition 2.5. Let $S$ be a real Riemann surface of genus $g=l-1$ with $l$ real branches (that is, $S$ is an M-curve) and $D$ be a real divisor of degree $l+2$ such that every real branch of $S$ contains an odd number of points from $D$ (counted with multiplicity). Then the algebraic curve $A=\iota_{D}(S) \subset \mathbb{P}^{3}$ corresponding to $(S, D)$ is an irreducible algebraic Hopf link. In particular, $A$ is non-singular, and $r(D)=3$.

Proof. The proposition is the special case of [Hui03, Corollary 2.8] for $r=3$.

COROLlary 2.6. Irreducible algebraic Hopf links of the same chirality are rigidly isotopic.

Proof. Let $L, L^{\prime} \subset \mathbb{R} \mathbb{P}^{3}$ be two irreducible algebraic Hopf links represented as $\iota_{D}(S)$ and $\iota_{D^{\prime}}\left(S^{\prime}\right)$ for real Riemann surfaces $S$ and $S^{\prime}$ and real divisor $D$ and $D^{\prime}$ on them. By Proposition 2.3, the Riemann surfaces $S$ and $S^{\prime}$ are $M$-curves. Therefore, their quotients by the complex conjugation are diffeomorphic to a disk with $g$ holes. Thus (see, for example, [SS89, Theorem 2.2]), the surfaces $S$ and $S^{\prime}$ can be deformed into each other through a family of real curves. 


\section{G. Mikhalkin and S. Orevkov}

Without loss of generality (by taking an appropriate real plane section of the corresponding irreducible algebraic Hopf links), we may assume that the real divisors $D$ and $D^{\prime}$ contain a single point on each real branch of $S$ and $S^{\prime}$ as well as a pair of complex conjugate points. Thus, we may enhance the deformation of $S$ to $S^{\prime}$ with a deformation of $D$ into $D^{\prime}$ in the space of real divisors on real Riemann surfaces. By Proposition 2.5, this deformation corresponds to a deformation in the class of irreducible algebraic Hopf links, that is, a rigid isotopy.

Therefore, $L$ and $L^{\prime}$ are rigidly isotopic up to a projective equivalence. Since the group $P G L_{4}(\mathbb{R})$ consists of two connected components, $L$ and $L^{\prime}$ are rigidly isotopic if and only if their invariant $w_{\lambda}$ (which cannot vanish for a $M W_{\lambda}$-link) is of the same sign.

Remark 2.7. Note that Conjecture 3.4 of [Hui03] is false. Not only algebraic Hopf links, but all $M W_{\lambda}$-links are unramified in the sense of [Hui03] by condition (iii) ${ }^{t}$ of [MO19, Theorem 2]. Taking a real algebraic link $L \subset \mathbb{R P}^{3}$ isotopic to $W_{g}\left(a_{0}, \ldots, a_{g}\right)$ with even $a_{j}$, we get an unramified curve whose real branches are contractible in $\mathbb{P}^{3}$. The existence of such a link is ensured by [MO19, Theorem 3].

\section{Nodal links}

Recall that a nodal projective curve $A \subset \mathbb{P}^{n}$, for $n \geqslant 2$, is a (complex) algebraic curve such that all of its singularities are simple nodes, that is, crossing points of two non-singular local branches with distinct tangent lines. As before, $A$ is real if it is invariant with respect to the involution of complex conjugation $\mathbb{P}^{n} \rightarrow \mathbb{P}^{n}$. The real locus $\mathbb{R} A=\mathbb{R} \mathbb{P}^{n} \cap A$ of a nodal curve is a disjoint union of immersed circles and a finite set $\mathbb{R} E \subset \mathbb{R} A$. The points of $\mathbb{R} E$ are called solitary nodes of $\mathbb{R} A$.

DeFinition 3.1. A subset $L \subset \mathbb{R P}^{3}$ is called an irreducible nodal real algebraic link if it is infinite and there exists an irreducible nodal real algebraic curve $A \subset \mathbb{R} \mathbb{P}^{3}$ such that $L=\mathbb{R} A$ and $L$ is not contained in any proper projective subspace of $\mathbb{R P}^{3}$. In particular, $L \neq \emptyset$. Two nodal real algebraic links are rigidly isotopic if they can be connected with a 1-parametric isotopy in the class of nodal real algebraic links of the same degree and with the same number of nodes.

A node of $L$ is a node of the curve $A$.

Nodal real algebraic links provide a generalization of real algebraic links. As in the case of real algebraic links, an irreducible nodal real algebraic link $L \subset \mathbb{R} \mathbb{P}^{3}$ uniquely determines $A \subset \mathbb{P}^{3}$.

DEFINITION 3.2. We say that a real algebraic link $L_{1}$ degenerates to a real nodal algebraic link $L_{0}$ if there exists a continuous family of real algebraic links $L_{t} \subset \mathbb{R P}^{3}$, for $t \in[0,1]$, of constant degree such that $L_{t}$ for $t>0$ is a (non-nodal) real algebraic link. In this case, we also say that the nodal link $L_{0}$ can be perturbed to the smooth link $L_{1}$. The perturbation or degeneration is called equigeneric if the genus stays constant.

A smooth perturbation of a nodal curve is equigeneric if each node is perturbed as in Figure 2 (left) but not as in Figure 2 (right). All degenerations and perturbations considered in this paper are assumed to be equigeneric.

Proposition 3.3. Any irreducible real algebraic link $L=\mathbb{R} A \subset \mathbb{R P}^{3}$ of genus $g$ and degree $d$ degenerates to a nodal link with at least $d-g-3$ nodes.

Proof. By induction, it is enough to assume that if $L=\mathbb{R} A$ is a nodal link of genus $g$ and degree $d$ with $n<d-g-3$ nodes, then it degenerates to a nodal link with at least one node more. 


\section{RIGID ISOTOPY OF MAXIMALLY WRITHED LINKS}

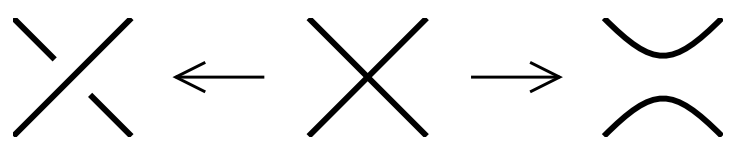

FIGURE 2. Equigeneric (on the left) and non-equigeneric (on the right) perturbations of a spatial nodal curve

Let $p \in \mathbb{R P}^{3}$ be a generic point such that the image $B=\pi_{p}(A) \subset \mathbb{P}^{2}$ under the projection $\pi_{p}: \mathbb{P}^{3} \backslash\{p\} \rightarrow \mathbb{P}^{2}$ is a real nodal planar curve. By choosing $p$ on a line passing through two distinct real points of $A$, we may assume that $B$ has a real node with two real branches which is not a projection of a node of $A$. This means that there are distinct points $x, y \in \mathbb{R} A$ such that

$$
u=\pi_{p}(x)=\pi_{p}(y)
$$

We choose coordinates such that $p=(0: 0: 0: 1)$.

Let $\iota: S \rightarrow A$ be the normalization and $D=\iota^{-1}(A \cap H)$ be the divisor on $S$ obtained as the intersection of $A$ and a generic real plane $H \subset \mathbb{P}^{3}$. The divisor $D$ is a real divisor of rank $r \geqslant d-g$. The immersions of $S$ given by $A \subset \mathbb{P}^{3}$ and $B \subset \mathbb{P}^{2}$ correspond to a real projective 3-dimensional subspace of $|D|$ and a real projective plane inside it.

Consider the vector space $\Gamma(S, D)$ formed by the sections of the line bundle defined by $D$. In the coordinates, the curve $A \subset \mathbb{P}^{3}$ is given by four linearly independent sections $s_{0}, s_{1}, s_{2}, s_{3} \in$ $\Gamma(S, D)$ such that $s_{0}, s_{1}, s_{2}$ define the curve $B \subset \mathbb{P}^{2}$. The condition that $s_{0}, s_{1}, s_{2}, s$ define an immersion with $n$ prescribed nodes imposes $n$ linear conditions on $s \in \Gamma(S, D)$. Let $V \subset \Gamma(S, D)$ be the space of all sections satisfying these conditions; in particular, we have $s_{3} \in V$. Since $r(D)-n>3$, it follows that there exists an $s_{4} \in V$ not contained in the linear span of $s_{0}, s_{1}$, $s_{2}, s_{3}$. Taking $s_{4}$ close to $s_{3}$, we may assume that $s_{0}, s_{1}, s_{2}, s_{4}$ define a curve $A^{\prime} \subset \mathbb{P}^{3}$ without a node at $\pi_{p}^{-1}(u)$.

With the help of the plane sections of the curves $A$ and $A^{\prime}$ passing through one point of intersection with $\pi_{p}^{-1}(u)$ but not the other, we can choose $s_{j}^{\prime} \in \Gamma(D, S)$, for $j=3$, with the following properties:

(1) $s_{j}$ is a linear combination of $s_{0}, s_{1}, s_{2}, s_{j}^{\prime}$ (in particular, the curve defined by $s_{0}, s_{1}, s_{2}, s_{3}^{\prime}$ is projectively equivalent to $A$ );

(2) $s_{3}^{\prime}(\tilde{x})=0=s_{4}^{\prime}(\tilde{y})$;

(3) $s_{3}^{\prime}(\tilde{y}) \neq 0 \neq s_{4}^{\prime}(\tilde{x})$.

Here $\tilde{x}=\iota^{-1}(x), \tilde{y}=\iota^{-1}(y) \in S$. Note that property (1) implies the linear independence of $s_{0}$, $s_{1}, s_{2}, s_{3}^{\prime}, s_{4}^{\prime}$.

The image of $S$ in $\mathbb{P}^{3}$ under $\left[s_{0}: s_{1}: s_{2}: s_{3}^{\prime}+t s_{4}^{\prime}\right]$, for $t \in \mathbb{R}$, is a real curve $A_{t} \subset \mathbb{P}^{3}$ with $A_{0}=A$. Since the first three sections define a nodal immersion to $\mathbb{P}^{2}$, all singularities of $A_{t} \subset \mathbb{P}^{3}$ (if any) are nodes. By the linear independence of $s_{0}, s_{1}, s_{2}, s_{3}^{\prime}, s_{4}^{\prime}$, the curve $A_{t}$ cannot be contained in a plane in $\mathbb{P}^{3}$.

By properties (2) and (3), there exists a $t_{u} \in \mathbb{R}$ such that $A_{t_{u}}$ has a node over $u$ while $A_{0}$ does not have a node over $u$. Thus, $A$ can be degenerated to a curve with at least one node (though not necessarily over $u$ since another node may appear during the deformation of $t$ from 0 to $t_{u}$; in particular, the new node could be solitary or even a pair of complex conjugate nodes). 


\section{G. Mikhalkin and S. Orevkov}

\section{Nodal $M W_{\lambda}$-links and chord diagrams}

Definition 4.1. An irreducible nodal algebraic link is called a nodal $M W_{\lambda^{-}}$link if it can be (equigenerically) perturbed to a (smooth) $M W_{\lambda}$-link.

Lemma 4.2. All nodes of a nodal $M W_{\lambda}$-link are real and non-solitary.

Proof. Let $L_{0}$ be a nodal $M W_{\lambda}$-link and $L_{t}$, for $t \in(0,1]$, be smooth $M W_{\lambda}$-links degenerating to $L_{0}$. If $L_{0}$ has a non-real node $\nu$, then the planar curve $\pi_{p}\left(L_{\epsilon}\right) \subset \mathbb{P}^{2}$ must have a non-real node near $\pi_{p}(\nu)$ for small $\epsilon>0$. Thus, $L_{\epsilon}$ cannot be maximally writhed.

Suppose that $L_{0}=\mathbb{R} A_{0}$ has a solitary real node $\nu$. Let $p \in \mathbb{R} \mathbb{P}^{3} \backslash \mathbb{R} A_{0}$ be a point on a line $\ell$ connecting a pair of complex conjugate points of $A_{0}$. The projection $\pi_{p}\left(A_{\epsilon}\right) \subset \mathbb{P}^{2}$ has two solitary nodal points: one corresponding to $\nu$ and one corresponding to $\ell$. We get a contradiction to condition $\left(\mathrm{iv}^{a}\right)$ of $[\mathrm{MO} 19$, Theorem 2].

Many of the properties of $M W_{\lambda}$-links found in [MO19] also hold for any nodal $M W_{\lambda}$-link $L_{0}=\mathbb{R} A_{0} \subset \mathbb{R} \mathbb{P}^{3}$.

Let $q \in A_{0} \backslash \mathbb{R} A_{0}$. Denote by $\ell_{q \bar{q}} \subset \mathbb{R P}^{3}$ the real line whose complexification in $\mathbb{P}^{3}$ passes through $q$ (and thus also through $\bar{q}$ ).

Lemma 4.3. For any $q \in A_{0} \backslash \mathbb{R} A_{0}$, we have $\ell_{q \bar{q}} \cap L_{0}=\emptyset$.

Proof. By Lemma 4.2, the nodal link $L_{0}$ has no solitary nodes. Thus, at a point of $\ell_{q \bar{q}} \cap L_{0}$, there exists a tangent plane $H \subset \mathbb{R} \mathbb{P}^{3}$ containing $\ell_{q \bar{q}}$. The existence of such a plane contradicts the property [MO19, Theorem 2(ii)] of $M W_{\lambda}$-links which is clearly conserved under passing to the limiting nodal links.

COROLlary 4.4. For each real branch $K$, the linking number

$$
a(K)=2\left|\operatorname{lk}\left(\ell_{q \bar{q}}, K\right)\right|
$$

(taken twice) in $\mathbb{R P}^{3}$ is independent of $q \in A_{0} \backslash \mathbb{R} A_{0}$.

Proof. Lemma 4.3 implies the corollary since the curve $A_{0}$ is irreducible, and thus the family of lines of the form $\ell_{q \bar{q}}$, for $q \in A_{0} \backslash \mathbb{R} A_{0}$, is connected.

We have

$$
\sum_{K} a(K)=d-2
$$

by [MO19, equation (3)] as the integer numbers $a(K)$ agree with the numbers introduced in [MO19, Lemma 4.12] under the degeneration of $L_{t}$ to $L_{0}$.

When speaking of nodal curves, we shall use the language of chord diagrams.

Definition 4.5. A chord diagram $(X, \Lambda)$ is a pair consisting of the disjoint union $\Lambda$ of $l$ oriented circles and the topological space $X$ obtained as the result of attachment of $\delta \geqslant 0$ intervals (chords) along their endpoints to $\Lambda$ so that distinct endpoints are glued to distinct points of $\Lambda$. We consider $(X, \Lambda)$ up to homeomorphisms of the pair preserving the orientation of $\Lambda$. We denote the complement of the chord endpoints in $\Lambda$ by $\Lambda^{\circ} \subset \Lambda$.

Definition 4.6. A chord diagram $(X, \Lambda)$ is called planar if it can be embedded in the disjoint union $\Delta$ of $l$ copies of 2-disks in such a way that $\Lambda$ is mapped to the boundary of $\Delta$. 


\section{RIGID ISOTOPY OF MAXIMALLY WRITHED LINKS}

Clearly, no chord of a planar chord diagram can connect points from different components of $\Lambda$. Thus, a planar diagram for arbitrary $l$ is a disjoint union of $l$ connected planar chord diagrams.

By a loop $\lambda$ in a chord diagram $(X, \Lambda)$, we mean a simple closed loop in $X$. If $(X, \Lambda)$ is planar and $j: X \rightarrow \Delta$ is an embedding from Definition 4.6, then for each component of $\Delta \backslash j(X)$, there is a loop in $X$ around this component; we refer to these loops as planar loops.

Definition 4.7. The chord diagram $X\left(L_{0}\right)$ of a nodal link $L_{0}$ is the chord diagram obtained by attaching a chord to $\mathbb{R} \tilde{A}_{0}$ connecting the respective points of the normalization for every node of $\mathbb{R} A_{0}$.

For a loop $\lambda \subset L_{0}$, the number

$$
a(\lambda)=2\left|\operatorname{lk}\left(\lambda, \ell_{q \bar{q}}\right)\right|
$$

is independent of the choice of $q \in A_{0} \backslash \mathbb{R} A_{0}$ by Lemma 4.3 and the irreducibility of $A_{0}$ (cf. Corollary 4.4).

Proposition 4.8. The chord diagram of a nodal $M W_{\lambda}$-link $L_{0}$ is planar.

Proof. Let $L_{0}=\mathbb{R} A_{0}$ be a nodal $M W_{\lambda}$-link and $\nu: \tilde{A}_{0} \rightarrow A_{0}$ be the normalization of $A_{0}$. Let $x$ be a node of $L_{0}$ and $H$ be the real plane tangent to both local branches of $A_{0}$ at $x$. Consider the intersection number $n(K)$ of $H$ and $A_{0}$ along a real branch $K \ni x$. Namely, $n(K)$ is the intersection number in $\mathbb{P}^{3}$ of the complexification of $H$ and $\nu(U)$, where $U$ is a small neighborhood of $\tilde{K}$ in $\tilde{A}_{0}$ (here $\tilde{K}$ is the connected component of $\mathbb{R} \tilde{A}_{0}$ such that $\nu(\tilde{K})=K$ ). Consider a point $q \in \nu(U) \backslash K$ close to $x$. Then the line $\ell_{q \bar{q}}$ is close to the tangent line at $x$ to $K$. The plane $H$ may be perturbed to a real plane $H^{\prime} \supset \ell_{q \bar{q}}$. We have $\#\left(H^{\prime} \cap K\right) \geqslant 2\left|\operatorname{lk}\left(\ell_{q \bar{q}}, K\right)\right|=a(K)$ since $H^{\prime} \supset \ell_{q \bar{q}}$. But, in addition, the complexification of $H^{\prime}$ intersects $A_{0}$ at $q$ and $\bar{q}$. Thus, $n(K) \geqslant \#\left(H^{\prime} \cap K\right)+2 \geqslant a(K)+2$.

If two distinct real branches $K_{1}$ and $K_{2}$ meet at $x$, then taking the sum over all real branches, we get the inequality

$$
\sum n(K) \geqslant \sum a(K)+4=d+2
$$

contradicting the Bezout theorem for the intersection of $H$ and $A_{0}$.

A node $y \in L_{0}$ where both local branches correspond to the same real branch $K$ defines a subdivision of $K$ into two loops $\lambda_{j} \subset K$, for $j=1,2$. Each loop $\lambda_{j}$ is the closure of the image of an arc of $\tilde{K} \backslash \nu^{-1}(y)$ under $\nu$. If the chord diagram $X\left(L_{0}\right)$ is not planar, then we may assume that $K, x, y$ are chosen so that $\lambda_{1}$ and $\lambda_{2}$ pass through $x$. We have $a\left(\lambda_{1}\right)+a\left(\lambda_{2}\right)=a(K)$; see (4.3).

Denote by $n\left(\lambda_{j}\right)$ the intersection number of the complexification of $H$ and $\nu\left(U_{j}\right)$ for a small neighborhood $U_{j}$ of $\nu^{-1}\left(\lambda_{j}\right)$ in $\tilde{A}_{0}$. We get $n\left(\lambda_{j}\right) \geqslant a\left(\lambda_{j}\right)+2$, as above. If $y \notin H$, then $\nu\left(U_{1} \cap U_{2}\right)$ is disjoint from $H$; thus, $n(K)=n\left(\lambda_{1}\right)+n\left(\lambda_{2}\right) \geqslant a(K)+4$, and we get a contradiction to the Bezout theorem as before.

If $y \in H$, then $n(K)=n\left(\lambda_{1}\right)+n\left(\lambda_{1}\right)-n(y)$, where $n(y)$ is the local intersection number of $A_{0}$ and the complexification of $H$ at $y$. But in this case, we have $n\left(\lambda_{1}\right)+n\left(\lambda_{1}\right) \geqslant a(K)+4+n(y)$, so we get a contradiction to the Bezout theorem anyway.

Definition 4.9. The degree-chord diagram of a nodal $M W_{\lambda}$-link $L_{0}$ is a chord diagram enhanced with a number $a(\lambda)$ defined for any planar loop of $L_{0}$. The number $a(\lambda)$ is the degree of the planar loop $\lambda$ of the planar chord diagram $X\left(L_{0}\right)$. 


\section{G. Mikhalkin and S. Orevkov}

\section{Divisors on singular Riemann surfaces}

Let $S$ be a (closed, connected, irreducible) Riemann surface.

Definition 5.1. A chord diagram $X_{S}$ on $S$ is a topological space obtained by attaching to $S$ a finite number of 1-cells (chords) $I \approx[0,1]$ in an acyclic way, that is, so that the union of all chords is a forest (in particular, no chord is a loop in $X_{S}$ ). Let $\delta$ be the number of chords. The singular surface $\Sigma=\Sigma\left(X_{S}\right)$ is obtained by contracting each chord to a point.

If the boundaries of the chords are disjoint in $S$, we say that $X_{S}$ is nodal. Then we may consider $\Sigma$ as an abstract nodal curve. Otherwise, $\Sigma$ has $k$-fold points with $k>2$.

The theory of divisors and their linear systems on $\Sigma$ is very similar to its counterpart on ordinary Riemann surfaces. Denote by $N \subset \Sigma$ the singular locus, that is, the image of the union of chords under the contraction map $c: X_{S} \rightarrow \Sigma$. Let $\tilde{N}=c^{-1}(N) \cap S$. As usual, for a meromorphic function $f: S \rightarrow \mathbb{P}^{1}=\mathbb{C} \cup\{\infty\}$, we denote by $(f)$ the divisor on $S$ composed of its zeroes and poles with multiplicities. Let $\Sigma^{*}=\Sigma \backslash N=S \backslash \tilde{N}$.

Let us fix an effective divisor $D=\sum a_{j} p_{j}$, with $a_{j} \in \mathbb{Z}_{>0}$ and $p_{j} \in S \backslash \tilde{N}$. The number $n=\sum a_{j}$ is the degree of $D$. Denote by $V_{D}$ the set of meromorphic functions $f: S \rightarrow \mathbb{P}^{1}$ such that $(f) \geqslant-D$ (that is, $f$ has, at worst, a pole of order $a_{j}$ at $p_{j}$ and is holomorphic elsewhere) and

$$
c(x)=c(y) \Longrightarrow f(x)=f(y) \quad \forall x, y \in S .
$$

The space $V_{D}$ is a vector space. We say that a divisor $D^{\prime}$ on $S$ is $\Sigma$-equivalent to $D$, and write $D \sim D^{\prime}$, if $D-D^{\prime}=(f)$ for $f \in V_{D}$. Recall that we require $D$ to be disjoint from $\tilde{N}$ while we do not impose this requirement on $D^{\prime}$. In particular, the relation $\sim$ is not an equivalence relation (cf. Remark 5.5). Let $|D|_{\Sigma}$ be the set of effective divisors $\Sigma$-equivalent to $D$. It can be naturally identified with the projectivization of $V_{D}$.

The Riemann-Roch theorem implies that for each chord $I \subset X_{S}$, there exists a meromorphic form $\omega_{I}$ on $S$ holomorphic on $S \backslash \partial I$ and having a pole of the first order at each point of $\partial I$. Clearly, the residues of $\omega_{I}$ at the two points of $\partial I$ must be opposite. Multiplying this form by a constant, we may assume that the residues of $\omega_{I}$ at $\partial I$ are \pm 1 . Furthermore, for a choice of a system of $a$-cycles, that is, of $g$ simple loops $\alpha_{j}$, for $j=1, \ldots, g$, in $S$ such that $S \backslash \bigcup_{j=1}^{g} \alpha_{g}$ is a planar domain, we may assume

$$
\int_{\alpha_{j}} \omega_{I}=0
$$

by adding a holomorphic form on $S$.

Denote by $\Omega(\Sigma)$ the vector space generated by the holomorphic forms on $S$ and the forms $\omega_{I}$ for the chords $I \subset X_{S}$. This is the space of meromorphic forms on $S$ holomorphic on $S \backslash \tilde{N}$, having at worst poles of the first order at the points of $\tilde{N}$ and such that the sum of residues at all points of $\tilde{N}$ corresponding to the same point of $N$ vanishes. Thus, $\Omega(\Sigma)$ depends only on $\Sigma$ and not on a particular representation of $\Sigma$ by $X_{S}$. We have

$$
\operatorname{dim} \Omega(\Sigma)=g+\delta .
$$

We refer to the elements of $\Omega(\Sigma)$ as $\Sigma$-holomorphic forms.

Any element of $H_{1}\left(\Sigma^{*}\right)$ defines a functional on $\Omega(\Sigma)$ through integration. The Jacobian of $\Sigma$ is defined as the quotient

$$
\operatorname{Jac}(\Sigma)=\Omega^{*}(\Sigma) / H_{1}\left(\Sigma^{*}\right)
$$




\section{RIGID ISOTOPY OF MAXIMALLY WRITHED LINKS}

of the vector space dual to $\Omega(\Sigma)$ by the image of $H_{1}\left(\Sigma^{*}\right)$. It is a $(g+\delta)$-dimensional complex variety homeomorphic to $\left(S^{1}\right)^{2 g} \times\left(\mathbb{C}^{\times}\right)^{\delta}$. The divisor $D$ defines the Abel-Jacobi map

$$
\alpha_{D}: \operatorname{Sym}^{n}\left(\Sigma^{*}\right) \rightarrow \operatorname{Jac}(\Sigma)
$$

by associating with $D^{\prime}$ the functional $\omega \mapsto \int_{\Gamma} \omega$, where $\Gamma$ is a 1-chain in $\Sigma^{*}$ with $\partial \Gamma=D^{\prime}-D$.

The following theorem and corollary are variants of the classical Abel-Jacobi and RiemannRoch theorems for the case of singular curves. Their different versions can also be found in textbooks.

Theorem 5.2 (Abel-Jacobi theorem for $\Sigma$ ). For an effective divisor $D^{\prime}$ supported on $\Sigma^{*}$, we have $D^{\prime} \in|D|_{\Sigma}$ if and only if $\alpha_{D}\left(D^{\prime}\right)=0 \in \operatorname{Jac}(\Sigma)$.

Proof. Suppose $D^{\prime} \in|D|_{\Sigma}$, that is, $(f)=D^{\prime}-D$ with $f \in V_{D}$. By the conventional Abel-Jacobi theorem, we have $\int_{\Gamma} \omega=0$ for any holomorphic 1-form $\omega$ in $S$. Suppose that $I$ is a chord in $X_{S}$ and $x_{+}, x_{-} \in S$ are the endpoints of $I$. Let $\omega_{I}$ be the meromorphic form on $S$, holomorphic on $S \backslash\left\{x_{+}, x_{-}\right\}$, with simple poles of residue \pm 1 at $x_{ \pm}$and satisfying (5.1). Multiplying $f$ by a scalar, we may assume that $f\left(x_{+}\right), f\left(x_{-}\right) \in \mathbb{C}$ are non-real numbers. The following equality is known as a reciprocity law for the Abelian differentials of the third kind (cf., for example, [Dub86, Lecture 6, Lemma 3])

$$
\int_{\Gamma} \omega_{I}=\int_{\Gamma_{I}} \frac{d f}{f}
$$

Here $\Gamma=f^{-1}\left(\mathbb{R}_{\geqslant 0} \cup\{\infty\}\right)$ is oriented in such a way that $\partial \Gamma=D^{\prime}-D$, while $\Gamma_{I}$ is a path in $S$ from $x_{-}$to $x_{+}$disjoint from $\Gamma$. To prove (5.2), it suffices to integrate the 1 -form $\log (f) \omega_{I}$ over a small contour $\gamma$ going around $\Gamma$ for a holomorphic branch of $\log (f)$ in $S \backslash \Gamma$. Since the values of $\log (f)$ at the two sides of $\Gamma$ differ by $2 \pi i$, we see that $\int_{\gamma} \log (f) \omega_{I}$ equals $2 \pi i$ times the left-hand side of (5.2). By Cauchy's residue formula, $\int_{\gamma} \log (f) \omega_{I}$ equals $2 \pi i$ times the right-hand side of $(5.2)$.

Since $f \in V_{D}$, the right-hand side of (5.2) is zero modulo $2 \pi i \mathbb{Z}$, and thus $\alpha_{D}\left(D^{\prime}\right)=0$. Conversely, suppose that $\alpha_{D}\left(D^{\prime}\right)=0$. By the conventional Abel-Jacobi theorem, there exists a meromorphic function $f: S \rightarrow \mathbb{P}^{1}$ with $(f)=D^{\prime}-D$. By (5.2), the integral $\int_{\Gamma_{I}} \mathrm{~d} f / f$ vanishes (modulo $2 \pi i \mathbb{Z}$ ) for any chord $I$. Thus $f \in V_{D}$.

Denote by $\Omega_{-D}(\Sigma) \subset \Omega(\Sigma)$ the vector space formed by the $\Sigma$-holomorphic forms vanishing in all points of $D$.

Corollary 5.3 (Riemann-Roch theorem for $\Sigma$ ). We have

$$
\operatorname{dim}|D|_{\Sigma}=n-g-\delta+\operatorname{dim} \Omega_{-D}(\Sigma) .
$$

Proof. Consider the differential $d \alpha$ of the Abel-Jacobi map at $D \in \operatorname{Sym}^{n}\left(\Sigma^{*}\right)$. It is a linear map from the $n$-dimensional vector space composed as the direct sum of the tangent spaces of $S$ at the points of $D$ (with multiplicities) to the $(g+\delta)$-dimensional space $\Omega^{*}(\Sigma)$. The cokernel of this differential coincides with the space $V_{D}$. According to Theorem 5.2, the kernel is $V_{D}$.

We say that $D$ is non-special in $\Sigma$ if $\operatorname{dim}|D|_{\Sigma}=n-g-\delta$, that is, if $\Omega_{-D}(\Sigma)=0$ (recall that $\left.\operatorname{supp} D \subset \Sigma^{*}\right)$.

Consider the chord diagram $X_{S}^{I}$ obtained from $X_{S}$ by removing a chord $I$ and the corresponding singular surface $\Sigma^{I}$. 


\section{G. Mikhalkin and S. Orevkov}

Lemma 5.4. If a divisor $D=\sum_{j=1}^{n} a_{j} p_{j}$, with $p_{j} \in \Sigma^{*}$, is non-special in $\Sigma$, then it is also non-special in $\Sigma^{I}$.

Proof. By definition, we have $\Omega\left(\Sigma^{I}\right) \subset \Omega(\Sigma)$, whence $\Omega_{-D}\left(\Sigma^{I}\right) \subset \Omega_{-D}(\Sigma)$. Thus, the hypothesis of $\Omega_{-D}(\Sigma)=0$ implies the conclusion $\Omega_{-D}\left(\Sigma^{I}\right)=0$.

With each effective divisor $D$ such that $\operatorname{supp} D \subset \Sigma^{*}$, we associate the map

$$
\iota_{D}: S \rightarrow|D|_{\Sigma}^{\vee}
$$

sending $x$ to the hyperplane of $V_{D}$ corresponding to divisors $D^{\prime} \in|D|_{\Sigma}$ with $x \in D^{\prime}$. This mapping evidently factors through a mapping $\Sigma \rightarrow|D|_{\Sigma}^{\vee}$.

As in the classical theory, any holomorphic mapping $f: S \rightarrow \mathbb{P}^{k}$ which factors through $\Sigma$ is a composition of $\iota_{D}$ with a linear projection $\pi:|D|_{\Sigma}^{\vee} \rightarrow \mathbb{P}^{k}$, where $D$ is the pull-back of a generic plane section of $f(S)$. The image of $\pi$ is the minimal subspace of $\mathbb{P}^{k}$ containing $f(S)$.

Remark 5.5. When a smooth curve is embedded in a complete linear system $|D|$, the embedded curve can be completely recovered (up to a projective transformation) by any hyperplane section. Nevertheless, the situation is very different in the case of nodal curves. If a hyperplane section passes through some nodes, then it does not determine the embedding. This is the reason why we have supposed $\operatorname{supp} D \subset \Sigma^{*}$ in this section.

\section{Real singular Riemann surfaces}

A chord diagram $X_{S}$ on a Riemann surface $S$ is real algebraic if the Riemann surface $S$ is real (that is, endowed with an antiholomorphic involution conj: $S \rightarrow S$ ) and the boundaries of all chords are contained in $\mathbb{R} S=\operatorname{Fix}(S)$. Then we may consider

$$
\mathbb{R} X_{S}=X_{S} \backslash(S \backslash \mathbb{R} S)
$$

(the union of $\mathbb{R} S$ with all the chords). If $X_{S}$ is nodal, then the pair $\left(\mathbb{R} X_{S}, \mathbb{R} S\right)$ is the chord diagram in the sense of Definition 4.5. Otherwise, it can be considered as a degeneration of such a diagram.

We say that a real algebraic chord diagram $X_{S}$ is planar if $\mathbb{R} X$ can be embedded into the disjoint union of disks in such a way that $\mathbb{R} S$ is mapped homeomorphically onto the boundary. Note that in the case when the chord boundaries are disjoint, this definition agrees with Definition 4.6. We have $l+\delta$ planar loops in $\mathbb{R} X_{S}$ (here $l=b_{0}(\mathbb{R} S)$ ) that correspond to the components of the complement of the image of this embedding.

Lemma 6.1. Suppose that $X_{S}$ is a real planar chord diagram and $\omega \in \Omega(\Sigma)$ is a real form on $S$ whose zeroes are disjoint from $\tilde{N}$. Then every planar loop in $\mathbb{R} X_{S}$ contains an even number of zeroes of $\omega$ (counted with multiplicities).

Proof. In the complement of its zeroes, a real form $\omega$ defines an orientation of the underlying curve. Since the residues of $\omega$ at the endpoints of each chord are opposite, this orientation agrees with an orientation of the planar loop near the chord. Thus, the lemma follows from the orientability of a circle.

Corollary 6.2. An effective real divisor $D=\sum_{j=1}^{n} a_{j} p_{j}$, with $a_{j} \in \mathbb{Z}_{>0}$ and $p_{j} \in \Sigma^{*}$, is nonspecial if $X_{S}$ is planar and at least $g+\delta$ distinct planar loops of $X_{S}$ intersect $\operatorname{supp}(D)=\bigcup_{j=1}^{n}\left\{p_{j}\right\}$. 


\section{RIGID ISOTOPY OF MAXIMALLY WRITHED LINKS}

Proof. Suppose $\omega \in \Omega_{-D}(\Sigma)$. If $\omega$ does not have poles at the boundary of a chord in $X_{S}$, then we may remove this chord and the statement follows by induction from the corresponding statement for the resulting diagram in $(\delta-1)$ chords.

Otherwise, $\omega$ has a total of $2 g+2 \delta-2$ zeroes in $\Sigma^{*}$ (counted with multiplicities). But Lemma 6.1 implies the existence of at least $2 g+2 \delta$ zeroes contained in the given $g+\delta$ planar loops.

Consider a real algebraic nodal curve $A_{0} \subset \mathbb{P}^{3}$ endowed with a fixed orientation of its real branches and its real equigeneric perturbation $A_{t} \subset \mathbb{P}^{3}$, (cf. Definition 4.1 , but now $A_{t}$ is not required to be smooth) for small $t>0$. We say that $A_{t}$ is positive (respectively, negative) at a node $x \in A_{0}$ if the perturbed local branches form a positive (respectively, negative) crossing according to Figure 1 under a generic projection.

Note that the signs of $A_{t}$ at all nodes are invariant if we reverse the orientation of $A_{0}$. Thus we may choose any orientation in the case when $A_{0}$ is rational. In the case when $A_{0}$ is of type I (the case of this paper), we take a complex orientation of $\mathbb{R} A_{0}$.

Lemma 6.3. Let $\varphi: S \rightarrow \mathbb{P}^{3}$ be an analytic mapping which factors through an injective mapping $\Sigma \rightarrow \mathbb{P}^{3}$ and $D$ be the pull-back of a real plane section. Suppose that $A_{0}=\varphi(S)$ is a real nodal curve, $\operatorname{supp}(D) \subset \Sigma^{*}, D$ is non-special in $\Sigma$, and $\operatorname{dim}|D|_{\Sigma} \geqslant 3$. Let $I_{1}, \ldots, I_{k}$ be some chords of $X_{S}$.

Then for any orientation on $\mathbb{R} S$, there exists a real equigeneric perturbation $A_{t}$ of $A_{0}$ which has any prescribed signs at the nodes corresponding to $I_{1}, \ldots, I_{k}$ and which keeps all the other nodes.

Proof. Inductively, with the help of Lemma 5.4, it suffices to prove this lemma for $k=1$, that is, to prove that we may perturb a single node of $A_{0}$ equigenerically, keeping all the other nodes, and choosing any prescribed sign for the perturbation of this node. Since $A_{0} \subset \mathbb{P}^{3}$ is nodal, its projection onto $\mathbb{P}^{2}$ from a generic point of $\mathbb{P}^{3}$ is a planar nodal curve $B \subset \mathbb{P}^{2}$. Let $s_{0}, s_{1}, s_{2}, s_{3} \in V_{D}(\Sigma)$ be the sections defining the curve $A_{0}$ and be such that $s_{0}, s_{1}, s_{2}$ define the curve $B$ (cf. the proof of Proposition 3.3).

By Corollary 5.3, we have $\operatorname{dim} V_{D}\left(\Sigma^{\prime}\right)>\operatorname{dim} V_{D}(\Sigma)$, where $\Sigma^{\prime}$ is the singular surface corresponding to the diagram $X^{I_{1}}$, that is, when the chord $I_{1}$ is removed. Let $s \in V_{D}\left(\Sigma^{\prime}\right) \backslash V_{D}(\Sigma)$. Then $s_{0}, s_{1}, s_{2}, s$ define an analytic mapping $S \rightarrow \mathbb{P}^{3}$ that factor through $\Sigma^{\prime}$ but not through $\Sigma$. Thus, the $s_{0}, s_{1}, s_{2}, s_{3}+t s$ for $t \in \mathbb{R}$, define the required perturbation of $A_{0}$, where different signs of $t$ correspond to different signs of the node perturbation.

\section{Nodal Hopf links}

Definition 7.1. A nodal $M W_{\lambda}$-link is called a nodal Hopf link if it has $d-3-g$ nodes.

By (4.2), the degree of any planar loop in a nodal Hopf link is 1. Conversely, as the number of planar loops is $d-2$, we get the following characterization of nodal Hopf links.

Proposition 7.2. A nodal $M W_{\lambda}$-link $L_{0} \subset \mathbb{R P}^{3}$ is a nodal Hopf link if and only if the degree of each planar loop is 1.

The following proposition is an immediate consequence of Proposition 3.3.

Proposition 7.3. Any nodal $M W_{\lambda}$-link (in particular, a smooth $M W_{\lambda}$-link) can be degenerated to a nodal Hopf link. 


\section{G. Mikhalkin and S. Orevkov}

Lemma 7.4. Let $A$ be a real algebraic curve in $\mathbb{P}^{3}$. Let $P$ be a real plane such that $A \cap P$ is finite. Let $F \subset A \cap P$ be a finite set, and let $\lambda_{1}, \ldots, \lambda_{s}$ be loops contained in $\mathbb{R} A \backslash F$ such that all pairwise intersections $\lambda_{i} \cap \lambda_{j}$ are finite and each loop $\lambda_{i}$ is non-trivial in $H_{1}\left(\mathbb{R P}^{3}\right)$. Then

$$
\operatorname{deg} A \geqslant s+\sum_{x \in F}(A \cdot P)_{x},
$$

where $(A \cdot P)_{x}$ is the local intersection number of $A$ and $P$ at $x$.

Proof. It is enough to consider a perturbation $P^{\prime}$ of $P$ in the class of conjugation-invariant smooth 4-dimensional submanifolds of $\mathbb{P}^{3}$. We may choose $P^{\prime}$ to coincide with $P$ near $F$. In addition, we may ensure that $P^{\prime} \cap \mathbb{R} \mathbb{P}^{3}$ is transverse to each $\lambda_{j}$ and all local intersections of $P^{\prime}$ with $A$ are positive.

The following proposition generalizes Proposition 2.5. We use the notation of Section 5 . Let $V_{D}^{\vee}$ be the dual space of $V_{D}$ and $|D|_{\Sigma}^{\vee}$ be its projectivization.

Let $X_{S}$ be a planar, nodal, real algebraic chord diagram in $l=g+1$ circles and $\delta$ chords.

Definition 7.5. A Hopf divisor in $X_{S}$ is a real divisor $D \subset \Sigma^{*}$ of degree $l+\delta+2$ such that the part of $D$ contained in every planar loop of $X_{S}$ has odd degree.

In other words, $D$ has a single point at each of the $l+\delta$ planar loops of $X_{S}$, and also two more points which either form a complex conjugate pair or both belong to the same planar loop of $X_{S}$. Proposition 7.2 assures that a real plane section of a nodal Hopf link is a Hopf divisor. The next proposition assures the converse.

Recall that a real curve $A_{0} \subset \mathbb{P}^{3}$ is said to be hyperbolic with respect to a line $\ell \subset \mathbb{R P}^{3}$ if for any real plane $P \subset \mathbb{P}^{3}$ passing through $\ell$, each intersection point of $P \backslash \ell$ and $A_{0}$ is real.

Proposition 7.6. For a Hopf divisor $D \subset \Sigma^{*}$, the map

$$
\iota_{D}: S \rightarrow|D|_{\Sigma}^{\vee} \approx \mathbb{P}^{3}
$$

from (5.3) is a well-defined immersion whose image is a nodal Hopf link. Furthermore, $\iota_{D}$ factors through an embedding of $\Sigma$ into $|D|_{\Sigma}^{\vee}$.

Proof. Step 1: $\iota_{D}$ is an immersion to $\mathbb{P}^{3}$. We have $|D|_{\Sigma} \approx \mathbb{P}^{3}$ by Corollary 6.2 . The same corollary implies that $\operatorname{dim}|D|_{\Sigma}>|D-x|_{\Sigma}$ for any $x \in \operatorname{supp} D \cap \Sigma^{*}$. Also, we have

$$
\operatorname{dim}|D-\partial I|_{\Sigma^{I}}=\operatorname{dim}|D|_{\Sigma^{I}}-2<|D|_{\Sigma} .
$$

Here, the equality follows, once again, from Corollary 6.2 (recall that $X_{S}$ is planar, so both points of $\partial I$ belong to the same component of $\mathbb{R} S$ ), while the inequality follows from the observation that $|D|_{\Sigma}$ has only one additional linear constraint in addition to those of $|D|_{\Sigma^{I}}$. Thus, $\iota_{D}$ is well defined, and $A=\iota_{D}(S) \subset \mathbb{P}^{3}$ is a real algebraic curve of degree $l+\delta+2$ with plane sections parameterized by $|D|_{\Sigma}$.

If $\iota_{D}$ is not an immersion at $x \in S$, then any plane section containing $x$ has multiplicity greater than 1 at $x$. Taking the plane through $x$ and a pair of complex conjugate points of $A$, we get a contradiction with Lemma 7.4.

Step 2: $A$ is a nodal curve with exactly $\delta$ nodes. If $x \in \mathbb{R} A$ is such that $\iota_{D}^{-1}(x) \backslash \mathbb{R} S \neq \emptyset$, then the plane through $x$ and a pair of conjugate points of $A$ provides a contradiction with Lemma 7.4. If $x \in A \backslash \mathbb{R P}^{3}$ is such that $\#\left(\iota_{D}^{-1}(x)\right)>1$, then we get a similar contradiction by considering a real plane containing $x$ and $\operatorname{conj}(x)$. This means that $\left.\iota_{D}\right|_{S \backslash \mathbb{R} S}$ is an embedding. 


\section{RIGID ISOTOPY OF MAXIMALLY WRITHED LINKS}

Suppose that we have $\iota_{D}(x)=\iota_{D}(y)$ for $x \neq y \in \mathbb{R} S$. Consider the plane $H$ through $\iota_{D}(x)$ tangent to the local branches of $S$ at $x$ and $y$. The corresponding divisor has multiplicity at least 2 at $x$ and $y$. If $x, y \in \mathbb{R} S \backslash \tilde{N}$, then for parity reasons, there must be another intersection point of this plane with the planar cycles containing $x$ or $y$, which gives a contradiction. Suppose $y \in \tilde{N}$, and let $y^{\prime} \neq x$ be the other endpoint of the same chord. Then the divisor cut by $H$ also contains $y^{\prime}$. Let us perturb $H$ to a generic plane section with two imaginary points near $x$. By a degree count, no other imaginary points may appear under this perturbation. Thus, $y$ and $y^{\prime}$ produce at least three real intersection points which should be repartitioned among the two planar cycles adjacent to $I$. Thus, at least one planar cycle of $\mathbb{R} X_{S}$ contains more than a single point of the divisor. Since the divisor has a pair of complex conjugate points, we have a contradiction once again. Thus, $\iota_{D}$ identifies only the endpoints of the same chord.

To prove that $A$ is nodal, it remains to show that the two branches at each of its singular points are not tangent to the same direction. But if they were, we could find a plane tangent to one of the branch with order at least 3 and, simultaneously, tangent to the other branch with order of at least 2, and obtain a contradiction with Lemma 7.4.

Step 3: $A$ is hyperbolic with respect to any real tangent line (for a definition, see just before Proposition 7.6). Using Lemma 7.4, we conclude that a plane tangent to $\mathbb{R} A$ has only real intersections with $\mathbb{R} A$ (cf. condition (ii) in [MO19, Theorem 2]) and also that no plane may be tangent to a local branch of $\mathbb{R} A$ with order greater than 3 . The latter condition implies that the (differential geometric) torsion of $\mathbb{R} A$ cannot change sign within the same real branch of $A$. Reflecting the orientation of $\mathbb{R} \mathbb{P}^{3}$ if needed, we may assume that $\mathbb{R} A$ has points of positive torsion.

Step 4: The linking number of a pair of oriented tangent lines to $\mathbb{R} A$ is positive. Here we assume the orientation to be compatible with a fixed complex orientation of $\mathbb{R} A$. Due to the hyperbolicity of $\mathbb{R} A$ with respect to tangent lines, this fact follows from [MO19, Lemmas 4.6 and 4.7] (see, in particular, [MO19, Figure 2]). In turn, this positivity implies that all points of $\mathbb{R} A$ have positive torsion and also that all crossing points (in the knot-theoretic sense) of a plane projection of $\mathbb{R} A \subset \mathbb{R P}^{3}$ are positive.

Step 5: There exists a point $p \in \mathbb{R P}^{3}$ such that all singularities of $\pi_{p}(A)$ are ordinary real nodes with real local branches. The central projection $\pi_{p}: A \rightarrow \mathbb{P}^{2}$ from a generic real point $p$ on a tangent line $\ell$ to $\mathbb{R} A$ at its non-nodal point has a cusp corresponding to $\ell$. Furthermore, all singular points of the curve $\pi_{p}(\mathbb{R} A) \subset \mathbb{R P}^{2}$ must be real singularities such that all of their local branches are also real. Indeed, the curve $\pi_{p}(\mathbb{R} A)$ may not have a pair of complex conjugate singularities, as otherwise the inverse image under $\pi_{p}$ of the real line through this pair would intersect $A$ at least in four imaginary points, producing a contradiction with Lemma 7.4. Also, the curve $\pi_{p}(\mathbb{R} A)$ may not have a real singularity $q$ with imaginary branches as otherwise we would get a contradiction to Lemma 7.4 by considering the plane passing through $\ell$ and $\pi_{p}^{-1}(q)$.

Consider the central projection $\pi_{p^{\prime}}: A \rightarrow \mathbb{P}^{2}$ from a generic real point $p^{\prime}$ close to $p$. The image $\pi_{p^{\prime}}(A)$ is a real nodal curve with three types of nodes: the nodes of $A$, the perturbations of the nodes of $\pi_{p}(A)$, and the node resulting from the cusp of $\pi_{p}(A)$. Choosing $p^{\prime}$ in an appropriate way, we ensure that the last node has real local branches. Recall that all the nodes of $\pi_{p}(\mathbb{R} A)$ are positive as knot-theoretical crossing points by the previous step.

Step 6: $A$ is a nodal Hopf link. Lemma 6.3 allows us to perturb all the nodes of $\mathbb{R} A$ in a positive way. The result of the perturbation has $N_{d}-g$ positive crossings and thus is a $M W_{\lambda}$-link. 


\section{G. Mikhalkin and S. Orevkov}

Proposition 7.7. All nodal Hopf links of the same chirality (the sign of $w_{\lambda}$ for a smooth $M W_{\lambda^{-}}$ perturbation) and with homeomorphic chord diagrams are rigidly isotopic, that is, isotopic in the class of real nodal algebraic links of the same degree.

Proof. A nodal Hopf link is determined by a Hopf divisor on a planar real algebraic chord diagram by Proposition 7.6. If a homeomorphism between the chord diagrams of two nodal Hopf links respects the corresponding Hopf divisors, then there exists a 1-parametric family of Hopf divisors on planar real algebraic chord diagrams producing an isotopy between the two nodal Hopf link. Lemmas 7.8 and 7.9 reduce the general case to the case considered above.

Let $(X, \Lambda)$ be a planar chord diagram in $l$ circles with $\delta$ chords. We say that a triple $(X, \Lambda, \Delta)$ is a Hopf triple if $\Delta \subset \Lambda$ is a set of $\delta+l$ points disjoint from the chord endpoints and such that each planar loop of $(X, \Lambda)$ contains a single point of $\Delta$.

Let $I \subset X$ be one of the chords of $(X, \Lambda)$. The chord $I$ is adjacent to two plane loops, $\alpha_{1}^{I}$ and $\alpha_{2}^{I}$. Let $\Delta^{\prime} \subset X$ be a set of $l+\delta-2$ points disjoint from the chord endpoints and such that each planar loop of $(X, \Lambda)$ except for $\alpha_{1}^{I}$ and $\alpha_{2}^{I}$ contains a single point of $\Delta^{\prime}$.

Let $\Delta_{+}$(respectively, $\Delta_{-}$) be the union of $\Delta^{\prime}$ and the two-point set obtained by moving both points of $\partial I$ in the direction (respectively, contrary to the direction) of the orientation of $\Lambda$. Note that both $\left(X, \Lambda, \Delta_{+}\right)$and $\left(X, \Lambda, \Delta_{-}\right)$are Hopf triples. In this case, we say that these triples are linked with a chord move in $I$.

Lemma 7.8. For any two Hopf triples $\left(X, \Lambda, \Delta_{ \pm}\right)$linked with a chord move, there exist a nodal Hopf link $A_{0} \subset \mathbb{P}^{3}$ and two generic real planes $H_{ \pm} \in \mathbb{P}^{3}$ such that $\left(\mathbb{R} X_{A_{0}}, \mathbb{R} S, S \cap \mathbb{R} H_{ \pm}\right)$is homeomorphic to $\left(X, \Lambda, \Delta_{ \pm}\right)$. Here, $X_{A_{0}}$ is the natural chord diagram on the normalization $S$ of the nodal curve $A_{0}$.

Proof. Choose a real algebraic realization $\left(\mathbb{R} X_{S}, \mathbb{R} S, D^{\prime}\right)$ of $\left(X, \Lambda, \Delta^{\prime}\right)$. Define $D$ to be the divisor obtained as the union of $D^{\prime} \cup \partial I$ with a pair $\Pi$ of complex conjugate points in $S \backslash \mathbb{R} S$. Note that according to Lemma 6.2 , the divisor $D$ is non-special in the singular surface $\Sigma^{I}$ corresponding to the removal of $I$ from the chord diagram $X_{S}$. Thus, there exists a real divisor $E$ close to $D$ and $\Sigma^{I}$-equivalent to $D$ such that $E \cap \partial I=\emptyset$ and $\left(\mathbb{R} X_{S}, \mathbb{R} S, E \cap \mathbb{R} S\right)$ is a Hopf triple. Note that $D \in|E|_{\Sigma}$ since $D \in|E|_{\Sigma^{I}}$ and the value of a meromorphic function $f: S \rightarrow \mathbb{P}^{1}$ with $(f)=D-E$ is zero (and thus is the same) on both points of $\partial I$.

By Proposition 7.6, the image $A_{0}=\iota_{E}(S) \subset \mathbb{P}^{3}$ is a nodal Hopf link. Since $D \in|E|_{\Sigma}$, there exists a real plane $H \in \mathbb{P}^{3}$ with $\iota_{E}^{-1}(H)=D$. In particular, $H$ passes through the node of $A_{0}$ corresponding to $I$. Let $H_{ \pm}$be the real planes obtained by perturbing $H$ to two different sides of the node. Since the pencil of real planes through $\Pi$ defines a totally real map $S \backslash \Pi \rightarrow \mathbb{P}^{1}$ (that is, the inverse image of $\mathbb{R} \mathbb{P}^{1}$ coincides with $\left.\mathbb{R} S\right)$, the triples $\left(\mathbb{R} X_{A_{0}}, \mathbb{R} S, S \cap \mathbb{R} H_{ \pm}\right)$and $\left(X, \Lambda, \Delta_{ \pm}\right)$ are homeomorphic.

Lemma 7.9. Any two Hopf triples $\left(X, \Lambda, D_{1}\right)$ and $\left(X, \Lambda, D_{2}\right)$ on the same planar chord diagram $(X, \Lambda)$ can be linked with a sequence of chord moves.

Proof. Inductively on $\delta$, we may assume that the lemma holds for all planar chord diagrams in less than $\delta$ chords. Since $(X, \Lambda)$ is dual to a tree, there exists a chord $I$ dual to a leaf edge of the tree.

This means that there exists a planar loop $\alpha$ adjacent to $I$ and not adjacent to any other chord of $(X, \Lambda)$. We apply the induction hypothesis to the diagram obtained by removing $I$ from $(X, \Lambda)$ and the divisors obtained by removing $\alpha \cap D_{j}$ from $D_{j}$ for $j=1,2$. 


\section{RIGID ISOTOPY OF MAXIMALLY WRITHED LINKS}

Corollary 7.10. Two $M W_{\lambda}$-links of the same degree and chirality are rigidly isotopic if they can be degenerated to the nodal Hopf link with the same chord diagram.

Proof. To deduce this statement from Proposition 7.7, it suffices to show that there exists an open neighborhood of the map $\iota_{D}: S \rightarrow \mathbb{P}^{3}$ (with $\delta$ nodes in the image) in the space of all holomorphic maps $S \rightarrow \mathbb{P}^{3}$ of the same degree such that the space inside this neighborhood formed by the $M W_{\lambda}$-links is open and connected. Corollary 6.2 implies such connectedness once we perturb only one coordinate of $\mathbb{P}^{3}$, leaving the other coordinates (responsible for the planar diagram in $\mathbb{R P}^{2}$ ) unchanged. Combining this with the local connectedness of the space of perturbations of the planar diagram, we get the statement.

\section{Generalization and proof of Theorem 3}

We say that a degree-chord diagram $(X, \Lambda)$, see Definition 4.9 , is realized by an $M W_{\lambda}$-link $L_{0} \subset \mathbb{R P}^{3}$ if $X\left(L_{0}\right)$ is homeomorphic to $(X, \Lambda)$ as a chord diagram and a generic real plane section of $L_{0}$ containing a pair of complex conjugate point has $a(\lambda)$ points on each planar loop $\lambda$ in $X\left(L_{0}\right)$. Recall that $a(\lambda)$ does not depend on the choice of the plane section.

The following theorem generalizes Theorem 3 to nodal $M W_{\lambda}$-links. Thus, its proof also provides a proof of Theorem 3 .

Theorem 4. The nodal $M W_{\lambda}$-links of degree $d$ and genus $g$ are classified up to rigid isotopy by the degree-chord diagrams.

Namely, any planar degree-chord diagram $(X, \Lambda)$ in $l=g+1$ circles with $\delta$ chords and degree function on the planar loops satisfying (4.2) is realized by a nodal $M W_{\lambda}$-link of genus $g$ and degree $d$. Any two nodal $M W_{\lambda}$-links with the same degree-chord diagram and the same chirality (that is, the sign of $w_{\lambda}$ for its smooth $M W_{\lambda}$-perturbation) are rigidly isotopic.

Suppose that $(X, \Lambda)$ is a planar degree-chord diagram of a nodal Hopf link (that is, with the degree of each planar loop equal to 1$)$. Consider the diagram $(Y, \Lambda)$ obtained from $(X, \Lambda)$ by removing some chords. Then $(Y, \Lambda)$ is also a planar chord diagram. A planar loop $\lambda$ of $Y$ is composed of several planar loops of $X$. We set the degree of $\lambda$ to be the number of such planar loops in $X$.

LEMma 8.1. The degree-chord diagram $(Y, \Lambda)$ is realizable as the result of a perturbation of a nodal Hopf link with chord diagram $(X, \Lambda)$.

Proof. Consider a real algebraic chord diagram $X_{S}$ corresponding to $(X, \Lambda)$ and a divisor $D$ with a pair of complex conjugate points and a single non-nodal point at each of the planar loops of $X_{S}$. Let $\iota_{D}: S \rightarrow|D|_{\Sigma}^{\vee} \approx \mathbb{P}^{3}$ be the corresponding map (as in (5.3)). As in the proof of Proposition 7.6, by Corollary 6.2 and Lemmas 5.4 and 6.3, the nodes corresponding to the chords missing in $(Y, \Lambda)$ may be perturbed in a positive way, keeping the other nodes. The result is a nodal $M W_{\lambda}$-link $L_{Y}$ as it could be further perturbed to a smooth $M W_{\lambda}$-link. The number of points of $L_{Y}$ in its plane section near $D$ agrees with our definition of $a(\lambda)$.

Suppose that $(X, \Lambda)$ is a planar chord diagram, and let $x, y, z \in \Lambda$ be three distinct points different from the endpoints of the chords and contained in a single planar loop $\lambda$ of $X$ in the order agreeing with the cyclic orientation of $\lambda$. Consider a point $y^{\prime}$ on $\lambda$ close to $y$ and a point $z^{\prime}$ on $\lambda$ close to $z$ such that the cyclic order of $x, y, y^{\prime}, z, z^{\prime}$ agrees with that on $\lambda$. 


\section{G. Mikhalkin and S. Orevkov}
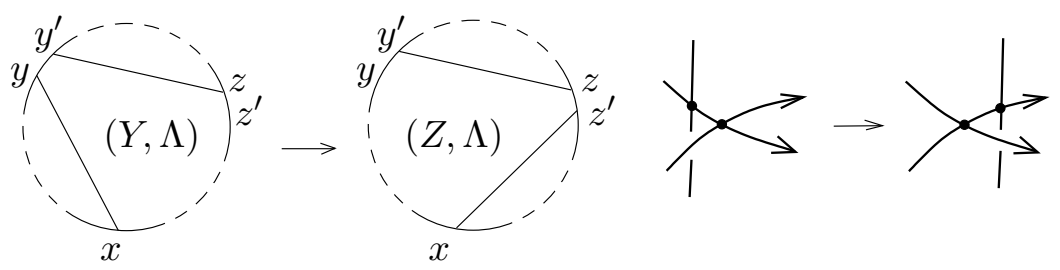

Figure 3 . The chord diagrams $(Y, \Lambda)$ and $(Z, \Lambda)$ and the resulting modification of nodal links

Form a planar diagram $(Y, \Lambda)$ by attaching two new chords to $X$ : the one connecting $x$ and $y$ and the one connecting $y^{\prime}$ and $z$; see Figure 3. Also form a planar diagram $(Z, \Lambda)$ by attaching two new chords to $X$ : the one connecting $x$ and $z^{\prime}$ and the one connecting $y$ and $z$. In this case, we say that the chord diagrams $(Y, \Lambda)$ and $(Z, \Lambda)$ differ by a chord slide.

Let $\left(X^{\prime}, \Lambda\right)$ be the plane diagram obtained from $(Y, \Lambda)$ by removing the chord $[x y]$ from $(Y, \Lambda)$ (which results in the same diagram as removing the chord $\left[x z^{\prime}\right]$ from $(Z, \Lambda)$ ). One of the planar loops of $X^{\prime}$ corresponds to two planar loops of $Y$ (or of $Z$ ). We set its degree equal to 2. All other planar loops have degree 1 . This turns $\left(X^{\prime}, \Lambda\right)$ into a degree-chord diagram. We consider the diagrams $(Y, \Lambda)$ and $(Z, \Lambda)$ as degree-chord diagrams with the degrees of all planar loops equal to 1.

Lemma 8.2. Suppose that two nodal $M W_{\lambda}$-links $L_{y}, L_{z} \subset \mathbb{R P}^{3}$ both have degree-chord diagram $\left(X^{\prime}, \Lambda\right)$ and degenerate to nodal Hopf links with chord diagrams $(Y, \Lambda)$ and $(Z, \Lambda)$. Then $L_{y}$ and $L_{z}$ are rigidly isotopic.

Proof. Choose a real Riemann surface $S$ of genus $g$ with $l=g+1$ real branches and an orientationpreserving homeomorphism between $\mathbb{R} S$ and $\Lambda$. Consider the real algebraic chord diagram $Y_{S}^{0}$ on $S$ obtained by attaching all chords of the diagram $(X, \Lambda)$, a chord connecting $x$ and $y$, and a chord connecting $y$ and $z$. Also consider the real algebraic chord diagram $Z_{S}^{0}$ on $S$ obtained by attaching all chords of the diagram $(X, \Lambda)$, a chord connecting $x$ and $z$, and a chord connecting $y$ and $z$. Note that both $Y_{S}^{0}$ and $Z_{S}^{0}$ are planar diagrams which are not nodal. Also note that $Y_{S}^{0}$ and $Z_{S}^{0}$ can be perturbed to nodal diagrams $Y_{S}$ and $Z_{S}$ as in Figure 3 .

Choose a divisor on $D$ of degree $l+\delta+2$ consisting of a pair of complex conjugate points on $S$ and a single non-nodal point at each planar cycle of $\mathbb{R} Y_{S}^{0}$. Note that then the same divisor will have a single point at each planar cycle of $\mathbb{R} Z_{S}^{0}$.

Real algebraic chord diagrams $Y_{S}^{0}$ and $Z_{S}^{0}$ define the same map $\iota_{D}: S \rightarrow|D|_{\Sigma} \approx \mathbb{P}^{3}$ since the linear system $|D|_{\Sigma}$ is the same for both diagrams: the functions in $V_{D}$ have the same values at $x, y$, and $z$. The image $A=\iota_{D}(S)$ has a triple point with three real branches at $\tau=\iota_{D}(x)=$ $\iota_{D}(y)=\iota_{D}(z)$; the rest of the singularities are nodes corresponding to the chords of $(X, \Lambda)$. This can be seen in the same way as in the proof of Proposition 7.6.

We claim that all three local branches of $A$ cannot be tangent to the same plane, as otherwise the corresponding plane section has a tangency of order 6 that must be repartitioned to the planar cycles. There are $l+g-3$ planar cycles which are not adjacent to the points $x, y, z \in Y_{S}^{0}$. As the degree of each of them is 1 and the degree of $A$ is $l+g+2$, we would get a contradiction. Thus, we may choose a generic point $p \in \mathbb{R P}^{3}$ such that the local intersection sign of the $y$-branch and the $z$-branch with the $x$-branch of $\pi_{p} \circ \iota_{D}(\mathbb{R} S) \subset \mathbb{R P}^{2}$ coincide; see Figure 4 . As in the proof of Proposition 7.6, we change the coordinates in $\mathbb{R P}^{3}$ so that $p=(0: 0: 0: 1)$. 


\section{RIGID ISOTOPY OF MAXIMALLY WRITHED LINKS}
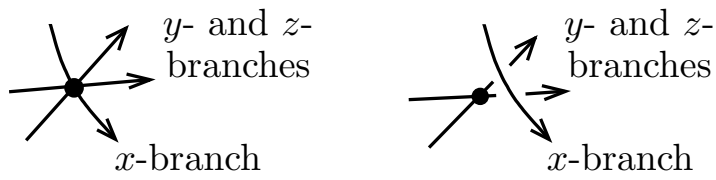

Figure 4. Perturbation of the triple point

Let $\Sigma^{\prime}$ be the singular surface obtained by contracting $S$ along each chord of $\left(X^{\prime}, \Lambda\right)$. Using Corollary 6.2 and Lemma 5.4 (cf. Lemma 6.3), we may perturb the map $\iota_{D}$, keeping the image of the curve under $\pi_{p}$ (the plane diagram), so that it factors through an embedding of $\Sigma^{\prime}$ and so that the new crossing points resulting from the perturbation are positive; see Figure 4. Performing the same perturbation for a family of chord diagrams connecting $Y_{S}^{0}$ to $Y_{S}$ and $Z_{S}^{0}$ to $Z_{S}$, we obtain an isotopy between $M W_{\lambda}$-links degenerating to the nodal Hopf links with the chord diagrams $(Y, \Lambda)$ and $(Z, \Lambda)$.

Proof of Theorem 4 . If we have two nodal $M W_{\lambda}$-links corresponding to the same planar degreechord diagram $(X, \Lambda)$, then both of them degenerate to nodal Hopf links as in Proposition 3.3. The chord diagrams of these nodal Hopf links must contain $(X, \Lambda)$ as a subdiagram, so that the degree of a planar cycle in $X$ equals the number of the planar cycles that appear in the subdivisions of this cycle in the larger diagrams. But then the two larger chord diagrams can be connected through a sequence of chord slides. Theorem 4 now follows from Lemma 8.2.

\section{REFERENCES}

Dub86 B. Dubrovin, Riemann surfaces and nonlinear equations (Moscow State Univ. Publ. House, Moscow, 1986).

Hui03 J. Huisman, Non-special divisors on real algebraic curves and embeddings into real projective spaces, Ann. Mat. Pura Appl. (4) 182 (2003), no. 1, 21-35; doi:10.1007/s10231-002-0053-1.

MO18 G. Mikhalkin and S. Orevkov, Topology of maximally writhed real algebraic knots, Doklady Akad. Nauk. 478 (2018), no 2, 141-144 (Russian), Doklady Math. 97 (2018), no. 1, 28-31 (English); doi:0.1134/S106456241801009X.

MO19 , Maximally writhed real algebraic links, Invent. Math. 216 (2019), no. 1, 125-152; doi: 10.1007/s00222-018-0844-7.

SS89 M. Seppälä and R. Silhol, Moduli spaces for real algebraic curves and real abelian varieties, Math. Z. 201 (1989), no. 2, 151-165; doi:10.1007/BF01160673.

Vir01 O. Viro, Encomplexing the writhe, in Topology, Ergodic Theory, Real Algebraic Geometry, Amer. Math. Soc. Transl. Ser. 2, vol. 202 (Amer. Math. Soc., Providence, RI, 2001), 241-256; doi: 10.1090/trans2/202/17.

Grigory Mikhalkin grigory.mikhalkin@unige.ch

Université de Genève, Mathématiques, Battelle Villa, 1227 Carouge, Suisse, Switzerland

Stepan Orevkov orevkov@math.ups-tlse.fr

Steklov Mathematical Institute, ul. Gubkina 8, 119991, Moscow, Russia,

IMT, Université Paul Sabatier, 118 route de Narbonne, 31062, Toulouse, France

AGHA Laboratory, Moscow Institute of Physics and Technology, Dolgoprudnyi, Russia 